\title{
Die Hartree-Fock-Theorie mit Zwischenzuständen: Klärung ihrer Struktur bei Zeitumkehrinvarianz und Anwendung auf das Kleinsche Rotations-Vibrationsmodell
}

\author{
A. Friederich und W. Gerling \\ Institut für Theoretische Kernphysik der Universität Bonn
}

Eingegangen am 22. Dezember 1975

\begin{abstract}
On Hartree-Fock Theory with Intermediate States:
Analysis of its Structure Using Time-Reversal Invariance and Application to Klein's Rotational-Vibrational Model
\end{abstract}

In an earlier paper we have extended the new Tamm-Dancoff method to the "New TammDancoff method with intermediate states". This extension makes it possible to treat the effect of nearby levels in many body systems with Green's functions. In addition to wellknown approximations, such as the Hartree-Fock theory and the Hartree-Bogoliubov theory, we obtain a series of new approximations. The "Hartree-Fock theory with intermediate states", which is the subject of the present investigation, is one of these. By using time reversal invariance we have succeeded in clarifying its structure, and we give the solution procedure. The exchange terms in the 1 -particle intermediate states can be represented by an additional potential $Y$, which (as is the case for the generalized density matrix $\rho$ ) has to be determined selfconsistently. In this way we have overcome the difficulties, that Kerman and Klein met in their "generalized Hartree-Fock approximation", which has some close similarities with our Hartree-Fock theory with intermediate states. We demonstrate our method for the exactly soluble rotational-vibrational model of Klein et al. Hereby we show how to treat conservation laws and the degeneracy of levels. The HartreeFock equations with three intermediate states turn out to give analytical expressions for the energies and the matrix elements. These agree excellently with the exact values in the rotational part of the spectrum.

\section{Einleitung}

Wenn mehrere Niveaus eines Vielteilchensystems dicht beieinander liegen oder sogar entartet sind, versagt die übliche, den Grundzustand auszeichnende Theorie der Green-Funktionen. An exakt lösbaren Modellen läßt sich leicht feststellen, daß auch die höheren Näherungen keine Änderung bringen, sondern da $\beta$ die Wirkungen benachbarter Niveaus von vornherein zu berücksichtigen sind [1]. Wir haben diese Forderung dadurch erfüllt, da $\beta$ wir an Stelle des Grundzustands einen mehrdimensionalen Unterraum des Hilbert-Raums setzen [2]. Wir nennen die $\mathrm{Zu}$ stände dieses Unterraums Zwischenzustände. Dabei haben wir die Gorkov-Faktorisierung der VierpunktGreen-Funktionen zum Vorbild genommen, wo die Grundzustände des $\mathscr{N}$ - und des $(x+2)$-TeilchenSystems als Zwischenzustände aufzufassen sind [3].
Durch sie wird das unendliche System der Bewegungsgleichungen für die Mehrpunkt-Green-Funktionen in ein Näherungssystem für "normale“ und „anomale“ Zweipunkt-Green-Funktionen, die Hartree-Bogoliubov-Theorie, übergeführt $[4,5]$. Unser Verfahren ist eine systematische Erweiterung:

1. Wir lassen als Zwischenzustände nicht nur einen $\mathscr{A}$ - und einen $(\mathfrak{N}+2)$-Teilchenzustand $z u$, wie in der Hartree-Bogoliubov-Theorie, sondern endlich viele beliebige, mit der Teilchenzahlerhaltung verträgliche. 2. Wir definieren auch die höheren Näherungen.

Wir brauchen nirgends vorauszusetzen, daß die Zwischenzustände eine Basis des ganzen Hilbert-Raums bilden.

Die Neue Tamm-Dancoff-Methode gibt einen Rahmen für weitere Verfeinerungen nicht-relativistischer 
und relativistischer Näherungsverfahren ab [6]. Die Idee ist, neue $n$-Punkt-Funktionen einzuführen, die nur von den Mehrpunkt-Green-Funktionen gleicher und niedrigerer Punktezahl abhängen, und das unendliche System der Bewegungsgleichungen durch Nullsetzen der neuen $n$-Punkt-Funktionen mit $n \geqq n_{0}$ und durch Vernachlässigen aller Bezeichnungen, in denen $n$-Punkt-Funktionen mit $n>n_{0}+1$ vorkommen, abzuschneiden. Die Gorkov-Faktorisierung ist ein Beispiel für $n_{0}=4$. Ohne die Methode der erzeugenden Funktionale wäre diese Idee undurchführbar. Daher formulieren wir Beziehungen zwischen den verschiedenen Sätzen von Mehrpunkt-Funktionen mit ihren erzeugenden Funktionalen. Dürr und Wagner haben vier unendliche Funktionensätze als Darstellung der Zustandsvektoren eingeführt, die $\tau$ (Mehrpunkt-Green-Funktionen), $\eta$-, $\zeta$ - und $\varphi$-Funktionen [7]. Bisher haben wir nur die Definition der $\eta$ und $\zeta$-Funktionen durch Einbeziehen einer beliebigen, aber endlichen Anzahl von Zwischenzuständen verallgemeinert [2]. Die $\eta$-Funktionen-Methode mit Zwischenzuständen liefert hermitische, doch nichtlineare Näherungssysteme für diese Zwischenzustände, während die $\zeta$-Funktionen-Methode mit Zwischenzuständen, auf den Ergebnissen der $\eta$-FunktionenMethode aufbauend, zu linearen, doch nicht-hermitischen Näherungssystemen für die anderen Zustände führt. In dieser Hinsicht unterscheiden sich $\eta$ - und $\zeta$ Funktionen-Methode mit Zwischenzuständen nicht von der gewöhnlichen $\eta$ - und der gewöhnlichen $\zeta$ Funktionen-Methode, die in erster Näherung die Hartree-Fock-Theorie bzw. die RPA ergeben [6]. Mit der Methode der erzeugenden Funktionale ist die Verallgemeinerung besonders einfach: Man ersetzt in der Arbeit von Dürr und Wagner [7] die erzeugenden Funktionale zwischen dem Grundzustand durch die Matrizen der erzeugenden Funktionale zwischen den Zwischenzuständen [2].

Bei der Faktorisierung der Vierpunkt-Green-Funktionen in Einpunkt-, Zweipunkt- und DreipunktGreen-Funktionen kommen $\mathscr{N}-,(\mathscr{N} \pm 1)$ - und $(\mathscr{N} \pm 2)$ Teilchenzustände als Zwischenzustände vor. Wir beschränken uns hier auf $m(0<m<\infty)$ Zwischenzustände der Teilchenzahl $\mathscr{N}$. Damit haben wir Einpunkt- und Dreipunkt-Green-Funktionen und Paarungsmatrixelemente eliminiert. Die Bewegungsgleichungen für die Mehrpunkt-Green-Funktionen gehen über in ein Näherungssystem für die ZweipunktGreen-Funktionen

$\left\langle I\left|T \Psi_{1} \Psi_{1}^{\dagger}\right| J\right\rangle ; \quad|I\rangle,|J\rangle \in\left\{\left|I_{1}\right\rangle,\left|I_{2}\right\rangle, \ldots,\left|I_{m}\right\rangle\right\}$.

Hier sind $\left|I_{1}\right\rangle,\left|I_{2}\right\rangle, \ldots,\left|I_{m}\right\rangle$ die Zwischenzustände und $\Psi_{i}$ der Feldoperator im Punkt

$i=\left(\mathrm{r}_{i}, t_{i}, \sigma_{i}, \tau_{i}\right)$.
Dieses Näherungssystem ist eine Verallgemeinerung der gewöhnlichen Hartree-Fock-Theorie, wo $m=1$ ist. Es ist von der Hartree-Bogoliubov-Theorie verschieden, da andere Übergangsmatrixelemente eingehen. Es soll Hartree-Fock-Theorie mit Zwischenzuständen heißen. Es ist das Anliegen dieser Arbeit, ihre Struktur aufzuklären. Das gelingt uns bei Zeitumkehrinvarianz, d.h. für den Fall, wo alle Matrixelemente des HamiltonOperators reell sind. A.K. Kerman and A. Klein stießen vor zehn Jahren auf ein ähnliches Gleichungssystem, das sie "generalized Hartree-Fock approximation (GHFA)" nannten [8-10]. Sie konnten jedoch seine Struktur nicht klären. An dieser Stelle macht sich der systematische Zugang über die Neue TammDancoff-Methode bezahlt: Die Hartree-Fock-Theorie mit Zwischenzuständen läßt eine hermitische Formulierung $\mathrm{zu}$, die wiederum die Formulierung eines Lösungsverfahrens ermöglicht. Es unterscheidet sich vom Lösungsverfahren der Hartree-Bogoliubov-Theorie [11] dadurch, daß die Austauschterme bezüglich der $\mathscr{N}$-Teilchen-Zwischenzustände durch ein Zusatzpotential $Y$ berücksichtigt werden, das ebenfalls selbstkonsistent bestimmt werden muß. Damit wird die Hartree-Fock-Theorie mit Zwischenzuständen durch doppelte Selbstkonsistenz gelöst: Selbstkonsistenz für die verallgemeinerte Dichtematrix $\rho_{I J}^{i i}=$ $\left\langle I\left|a_{i}^{\dagger}, a_{i}\right| J\right\rangle$ und Selbstkonsistenz für das Zusatzpotential $Y$. Mit der Klärung der Struktur steht uns ein weites Feld von Anwendungen offen: Rotationsbanden von Atomen, Molekülen und Kernen; Phasenübergänge in Vielteilchensystemen; Einbau von Erhaltungssätzen und Behandlung entarteter Niveaus. Wir demonstrieren die Struktur der Gleichungen, den Einbau von Erhaltungssätzen und die Behandlung entarteter Niveaus am Kleinschen Rotations-Vibrationsmodell $[12-14]$. Dabei sind die Hartree-FockGleichungen mit drei Zwischenzuständen (Grundzustand und zweifach entarteter, erster angeregter $\mathrm{Zu}$ stand) noch so einfach, daß man geschlossene Ausdrücke für Energien und Matrixelemente erhält. Leider beschreiben sie nur den Rotationsteil des Spektrums gut. Wir halten jedoch die gewonnenen Einsichten für lehrreich.

In Abschnitt 2 stellen wir die aus der $\eta$-Regel mit Zwischenzuständen folgenden Faktorisierungen der Vierpunkt-Green-Funktionen zusammen. Da die Faktorisierungsformeln bei $(\mathscr{N} \pm 1)$-Teilchen-Zwischenzuständen sehr lang sind, beschränken wir uns auf $\mathscr{N}$ - und $(\mathcal{N} \pm 2)$-Teilchen-Zwischenzustände. Wir betrachten nur einige Sonderfälle der zahlreichen Faktorisierungsmöglichkeiten, zu denen die Gorkov- und die zur Hartree-Fock-Theorie mit Zwischenzuständen führende Faktorisierung gehören. In Abschnitt 3 formulieren wir die Hartree-Fock-Theorie mit Zwischenzuständen für nicht-relativistische Vielteilchensysteme. 
In Abschnitt 4 klären wir ihre Struktur bei Zeitumkehrinvarianz. Diese Analyse liefert ein Lösungsverfahren. In Abschnitt 5 prüfen wir unser Lösungsverfahren am Kleinschen Rotations-Vibrationsmodell. In Abschnitt 6 diskutieren wir einige zukünftige Anwendungen unseres Verfahrens.

\section{Faktorisierung der Vierpunkt-Green-Funktionen bei.$V$ - und $(. V \pm 2)$-Teilchen-Zwischenzuständen}

In der vorhergehenden Arbeit haben wir ein allgemeines Schema zur Faktorisierung der MehrpunktGreen-Funktionen angegeben [2]. Wir stellen hier nur die aus der $\eta$-Regel mit Zwischenzuständen folgenden Faktorisierungsformeln für die Vierpunkt-GreenFunktionen zusammen, wobei wir der Einfachheit halber $(\mathscr{T} \pm 1)$-Teilchen-Zwischenzustände ausschlieBen. Darin ist die Faktorisierungsformel, die zur Hartree-Fock-Theorie mit Zwischenzuständen führt, bereits als Spezialfall enthalten.

Wir nehmen an, daß die zu den $\mathscr{A}$ - und $(\hat{A} \pm 2)$ Teilchen-Zwischenzuständen

$\left|I_{1}\right\rangle,\left|I_{2}\right\rangle, \ldots,\left|I_{m}\right\rangle \quad(0<m<\infty)$

gehörenden Vierpunkt-Green-Funktionen

$\left\langle I\left|T \Psi_{2} \Psi_{1} \Psi_{1}^{\dagger}, \Psi_{2}^{\dagger}\right| J\right\rangle$

$\left\langle I\left|T \Psi_{1} \Psi_{1}^{\dagger} \Psi_{2}^{\dagger}, \Psi_{3}^{\dagger}\right| J\right\rangle$

$\left\langle I\left|T \Psi_{3} \Psi_{2} \Psi_{1} \Psi_{1}^{\dagger}\right| J\right\rangle ; \quad|I\rangle,|J\rangle \in\left\{\left|I_{1}\right\rangle,\left|I_{2}\right\rangle, \ldots,\left|I_{m}\right\rangle\right\}$,

in die zu diesen Zwischenzuständen gehörenden Zweipunkt-Green-Funktionen

$\left\langle I\left|T \Psi_{1} \Psi_{1}^{\dagger}\right| J\right\rangle ;\left\langle I\left|T \Psi_{1}^{\dagger} \Psi_{2}^{\dagger} \cdot\right| J\right\rangle ;\left\langle I\left|T \Psi_{2} \Psi_{1}\right| J\right\rangle$,

faktorisiert werden. Wir haben folgende Faktorisierungen erhalten:

$$
\begin{aligned}
& \left\langle I_{i}\left|T \Psi_{2} \Psi_{1} \Psi_{1}^{\dagger} \cdot \Psi_{2}^{\dagger} \cdot\right| I_{j}\right\rangle \\
& =\frac{1}{2} \sum_{j_{1}=1}^{m}\left[\left\langle I_{i}\left|T \Psi_{1} \Psi_{1}^{\dagger}\right| I_{j_{1}}\right\rangle\left\langle I_{j_{1}}\left|T \Psi_{2} \Psi_{2}^{\dagger} \cdot\right| I_{j}\right\rangle\right. \\
& -\left\langle I_{i}\left|T \Psi_{2} \Psi_{1}^{\dagger}\right| I_{j_{1}}\right\rangle\left\langle I_{j_{1}}\left|T \Psi_{1} \Psi_{2}^{\dagger} \cdot\right| I_{j}\right\rangle \\
& -\left\langle I_{i}\left|T \Psi_{1} \Psi_{2}^{\dagger} \cdot\right| I_{j_{1}}\right\rangle\left\langle I_{j_{1}}\left|T \Psi_{2} \Psi_{1}^{\dagger} \cdot\right| I_{j}\right\rangle \\
& \left.+\left\langle I_{i}\left|T \Psi_{2} \Psi_{2}^{\dagger} \cdot\right| I_{j_{1}}\right\rangle\left\langle I_{j_{1}}\left|T \Psi_{1} \Psi_{1}^{\dagger} \cdot\right| I_{j}\right\rangle\right] \\
& +\frac{1}{2} \sum_{j_{1}=1}^{m}\left[\left\langle I_{i}\left|T \Psi_{2} \Psi_{1}\right| I_{j_{1}}\right\rangle\left\langle I_{j_{1}}\left|T \Psi_{1}^{\dagger} \cdot \Psi_{2}^{\dagger} \cdot\right| I_{j}\right\rangle\right. \\
& \left.+\left\langle I_{i}\left|T \Psi_{1}^{\dagger} \Psi_{2}^{\dagger} \cdot\right| I_{j_{1}}\right\rangle\left\langle I_{j_{1}}\left|T \Psi_{2} \Psi_{1}\right| I_{j}\right\rangle\right] ;
\end{aligned}
$$

$$
\begin{aligned}
& \left\langle I_{i}\left|T \Psi_{1} \Psi_{1}^{\dagger} \Psi_{2}^{\dagger}, \Psi_{3}^{\dagger}\right| I_{j}\right\rangle \\
& =\frac{1}{2} \sum_{j_{1} \cdots 1}^{m}\left[\left\langle I_{i}\left|T \Psi_{1}^{\dagger} \cdot \Psi_{2}^{\dagger}\right| I_{j_{1}}\right\rangle\left\langle I_{j_{1}}\left|T \Psi_{1} \Psi_{3}^{\dagger}\right| I_{j}\right\rangle\right. \\
& +\left\langle I_{i}\left|T \Psi_{2}^{\dagger} \Psi_{3}^{\dagger} \cdot\right| I_{j_{1}}\right\rangle\left\langle I_{j_{1}}\left|T \Psi_{1} \Psi_{1}^{\dagger}\right| I_{j}\right\rangle \\
& -\left\langle I_{i}\left|T \Psi_{1}^{\dagger} \cdot \Psi_{3}^{\dagger} \cdot\right| I_{j_{1}}\right\rangle\left\langle I_{j_{1}}\left|T \Psi_{1} \Psi_{2}^{\dagger} \cdot\right| I_{j}\right\rangle \\
& +\left\langle I_{i}\left|T \Psi_{1} \Psi_{1}^{\dagger} \cdot\right| I_{j_{1}}\right\rangle\left\langle I_{j_{1}}\left|T \Psi_{2}^{\dagger} \cdot \Psi_{3^{\prime}}^{\dagger}\right| I_{j}\right\rangle \\
& +\left\langle I_{i}\left|T \Psi_{1} \Psi_{3}^{\dagger} \cdot\right| I_{j_{1}}\right\rangle\left\langle I_{j_{1}}\left|T \Psi_{1}^{\dagger} \Psi_{2}^{\dagger}\right| I_{j}\right\rangle \\
& \left.-\left\langle I_{i}\left|T \Psi_{1}^{\dagger} \Psi_{2}^{\dagger} \cdot\right| I_{j_{1}}\right\rangle\left\langle I_{j_{1}}\left|T \Psi_{1}^{\dagger} \Psi_{3}^{\dagger}\right| I_{j}\right\rangle\right] ; \quad i, j=1, \ldots, m .
\end{aligned}
$$

Wenn man die Gleichungen (2.4) und (2.5) benutz1, die Vierpunkt-Green-Funktionen in den Bewegungsgleichungen zu faktorisieren, die Zweipunkt- und Vierpunkt-Green-Funktionen verbinden, erhält man neben vielen neuen Näherungen einige bekannte Spezialfälle:

(I) Hartree-Fock-Theorie: $m=1 ;\left|I_{1}\right\rangle=|0, \ldots\rangle$.

(II) Hartree-Bogoliubov-Theorie:

$\left.m=2 ;\left|I_{1}\right\rangle=|0, \cdot\rangle ;\left|I_{2}\right\rangle=|0,1\rangle+2\right\rangle$.

In diesem Rahmen ist auch die Hartree-Fock-Theorie mit Zwischenzuständen ein Spezialfall.

(III) Hartree-Fock-Theorie mit Zwischenzuständen: $\left|I_{1}\right\rangle,\left|I_{2}\right\rangle, \ldots,\left|I_{m}\right\rangle$ sind $m$ Zwischenzustände mit der Teilchenzahl . . Diese Zwischenzustände erlauben nur noch die Faktorisierung in die Zweipunkt-GreenFunktionen $\left\langle I\left|T \Psi_{1} \Psi_{1}^{\dagger}\right| J\right\rangle$. Bei nicht-relativistischen Vielteilchensystemen kann man außerdem den Grenzübergang

$t_{2}^{\prime}=2 \varepsilon ; t_{1}^{\prime}=\varepsilon ; t_{1}=-\varepsilon ; t_{2}=-2 \varepsilon ; \varepsilon>0 ; s \rightarrow 0$, machen. Damit nimmt die Gleichung (2.4) die Gestalt $\left\langle I_{i}\left|a_{i_{2}}^{\dagger} a_{i_{1}}^{\dagger} a_{i_{1}} a_{i_{2}}\right| I_{j}\right\rangle$

$=\frac{1}{2} \sum_{j_{1}=1}^{m}\left[\left\langle I_{i}\left|a_{i_{1}}^{\dagger} a_{i_{1}}\right| I_{j_{1}}\right\rangle\left\langle I_{j_{1}}\left|a_{i_{2}}^{\dagger} a_{i_{2}}\right| I_{j}\right\rangle\right.$

$-\left\langle I_{i}\left|a_{i_{1}}^{\dagger} a_{i_{2}}\right| I_{j_{1}}\right\rangle\left\langle I_{j_{1}}\left|a_{i_{2}}^{\dagger} a_{i_{1}}\right| I_{j}\right\rangle$

$-\left\langle I_{i}\left|a_{i_{2}}^{\dagger} a_{i_{1}}\right| I_{j_{1}}\right\rangle\left\langle I_{j_{1}}\left|a_{i_{1}}^{\dagger} a_{i_{2}}\right| I_{j}\right\rangle$

$\left.+\left\langle I_{i}\left|a_{i_{2}}^{\dagger} a_{i_{2}}\right| I_{j_{1}}\right\rangle\left\langle I_{j_{1}}\left|a_{i_{1}}^{\dagger} a_{i_{1}}\right| I_{j}\right\rangle\right] ; \quad i, j=1, \ldots, m$, (2.6) an. Gleichung (2.6) ist die für die Hartree-Fock-Theorie mit Zwischenzuständen grundlegende Faktorisierungsformel.

Zwar erhält sie die Symmetrie- und Hermitizitätseigenschaften der Vierpunkt-Funktion, aber für ein vollständiges System von Zwischenzuständen würde die rechte Seite nicht gleich der linken sein. Das ist nicht erstaunlich, da bei der Neuen Tamm-DancoffMethode die Näherungen durch Erhöhung der Punktezahl und der Anzahl der Zwischenzustände zu verbessern sind. Wir haben diesen einseitigen Grenzfall schon bei der Formulierung der $\eta$-Regel ausgeschlossen, um Konvergenzfragen aus dem Wege zu gehen. 


\section{Die nicht-relativistische Hartree-Fock-Theorie mit Zwischenzuständen}

Wir gelangen zur Hartree-Fock-Theorie mit Zwischenzuständen, wenn wir in den Bewegungsgleichungen die Vierpunkt-Funktionen $\left\langle I_{i}\left|a_{i_{2}}^{\dagger} a_{i_{1}}^{\dagger} a_{i_{1}} a_{i_{2}}\right| I_{j}\right\rangle ; i, j=$ $1, \ldots, m$, gemäß (2.6) faktorisieren. Dadurch entkoppeln die Vier- und Mehrpunkt-Funktionen von den Zweipunkt-Funktionen, so daß ein nicht-lineares Gleichungssystem für die Zweipunkt-Funktionen

$\left\langle I_{i}\left|a_{i_{1}}^{\dagger} a_{i_{1}}\right| I_{j}\right\rangle ; \quad i, j=1, \ldots, m$,

übrigbleibt. Dazu bilden wir die Matrixelemente des Kommutators $\left[H, a_{q}^{\dagger} a_{p}\right]$, wo

$H=\sum_{i_{1}, i_{1}} T^{i_{1} i_{1}} a_{i_{1}}^{\dagger} a_{i_{1}}+\frac{1}{4} \sum_{i_{2}, i_{1}, i_{1}, i_{2}} V^{i_{2} i_{1}, i_{2} i_{1}} a_{i_{2}}^{\dagger} a_{i_{1}}^{\dagger} a_{i_{1}} a_{i_{2}}$

der Hamilton-Operator ist, zwischen den Zuständen $\left|I_{1}\right\rangle, \ldots,\left|I_{m}\right\rangle$ :

$\left\langle I_{i}\left|\left[H, a_{q}^{\dagger} a_{p}\right]\right| I_{j}\right\rangle$

$=\sum_{i_{1}^{\prime}}\left\langle I_{i}\left|a_{i_{1}^{\prime}}^{\dagger} a_{p}\right| I_{j}\right\rangle T^{i_{1} q}+\frac{1}{2} \sum_{i_{2}^{\prime}, i_{1}^{\prime}, i_{1}} V^{i_{2} i_{1}^{\prime}, q i_{1}}\left\langle I_{i}\left|a_{i_{2}}^{\dagger} a_{i_{1}}^{\dagger} a_{i_{1}} a_{p}\right| I_{j}\right\rangle$

$-\sum_{i_{1}} T^{p i_{1}}\left\langle I_{i}\left|a_{q}^{\dagger} a_{i_{1}}\right| I_{j}\right\rangle-\frac{1}{2} \sum_{i_{1}, i_{1}, i_{2}} V^{p i_{1}, i_{2} i_{1}}\left\langle I_{i}\left|a_{q}^{\dagger} a_{i_{1}}^{\dagger} a_{i_{1}} a_{i_{2}}\right| I_{j}\right\rangle ;$

$i, j=1, \ldots, m$.

Wenn wir annehmen, daß die Zwischenzustände $\left|I_{1}\right\rangle, \ldots,\left|I_{m}\right\rangle$ als angenäherte Eigenzustände von $H$ nicht nur die Faktorisierungseigenschaft (2.6) haben, sondern auch Eigenzustände des „Quasiteilchenoperators" $h$ sind:

$h\left|I_{i}\right\rangle=\Omega_{i}\left|I_{i}\right\rangle ; \quad i=1, \ldots, m$,

der $H$ approximiert, erhalten wir die Hartree-FockTheorie mit Zwischenzuständen:

$$
\begin{aligned}
& 2\left(\Omega_{i}-\Omega_{j}\right)\left\langle I_{i}\left|a_{q}^{\dagger} a_{p}\right| I_{j}\right\rangle \\
& =\sum_{i_{1}^{\prime}}\left\langle I_{i}\left|a_{i_{1}^{\prime}}^{\dagger} a_{p}\right| I_{j}\right\rangle T^{i_{1} q} \\
& +\sum_{i_{2}, i_{1}, i_{1}} V_{i_{1}}^{i_{2} i_{1}, q i_{1}} \sum_{j_{1}=1}^{m}\left\langle I_{i}\left|a_{i_{2}}^{\dagger} a_{p}\right| I_{j_{1}}\right\rangle\left\langle I_{j_{1}}\left|a_{i_{1}}^{\dagger} a_{i_{1}}\right| I_{j}\right\rangle \\
& -\sum_{i_{1}} T^{p i_{1}}\left\langle I_{i}\left|a_{q}^{\dagger} a_{i_{1}}\right| I_{j}\right\rangle \\
& -\sum_{i_{1}, i_{1}, i_{2}} V^{p i_{1}^{\prime}, i_{2} i_{1}} \sum_{j_{1}=1}^{m}\left\langle I_{i}\left|a_{i_{1}}^{\dagger} a_{i_{1}}\right| I_{j_{1}}\right\rangle\left\langle I_{j_{1}}\left|a_{q}^{\dagger} a_{i_{2}}\right| I_{j}\right\rangle \\
& +\sum_{i_{1}^{\prime}}\left\langle I_{i}\left|a_{i_{1}}^{\dagger} a_{p}\right| I_{j}\right\rangle T^{i i_{1} a} \\
& +\sum_{i_{2}, i_{1}, i_{1}} V^{i_{2} i_{1}, q i_{1}} \sum_{j_{1}=1}^{m}\left\langle I_{i}\left|a_{i_{1}}^{\dagger} a_{i_{1}}\right| I_{j_{1}}\right\rangle\left\langle I_{j_{1}}\left|a_{i_{2}}^{\dagger} a_{p}\right| I_{j}\right\rangle \\
& -\sum_{i_{1}} T^{p i_{1}}\left\langle I_{i}\left|a_{q}^{\dagger} a_{i_{1}}\right| I_{j}\right\rangle \\
& -\sum_{i_{1}, i_{1}, i_{2}} V^{p i_{1}^{\prime}, i_{2} i_{1}} \sum_{j_{1}=1}^{m}\left\langle I_{i}\left|a_{q}^{\dagger} a_{i_{2}}\right| I_{j_{1}}\right\rangle\left\langle I_{j_{1}}\left|a_{i_{1}^{\prime}}^{\dagger} a_{i_{1}}\right| I_{j}\right\rangle ; \\
& \left\langle I_{i}|N| I_{i}\right\rangle=\mathscr{N} \text {; } \\
& i, j=1, \ldots, m \text {. }
\end{aligned}
$$

Wir müssen die Bewegungsgleichungen durch die Teilchenzahlbedingungen ergänzen, weil Zustände mit der Faktorisierungseigenschaft (2.6) im allgemeinen nicht Eigenzustände des Teilchenzahloperators $N$ sind. Wir bringen diese Gleichungen in eine übersichtlichere Form durch Einführung der verallgemeinerten Dichtematrix

$\rho_{A B}^{a b}=\left\langle A\left|a_{b}^{\dagger} a_{a}\right| B\right\rangle$

und der verallgemeinerten Hartree-Fock-Matrix

$$
\begin{aligned}
& \mathscr{H}_{A B}^{a b}=T^{a b} \delta_{A B}+\sum_{l, l^{\prime}} V^{a l^{\prime}, b l} \rho_{A B}^{l l}: \\
& 2\left(\Omega_{A}-\Omega_{C}\right) \rho_{A C}^{a c} \\
& =\sum_{k, K}\left(\rho_{A K}^{a k} \mathscr{H}_{K C}^{k c}-\mathscr{H}_{A K}^{a k} \rho_{K C}^{k c}+\rho_{K C}^{a k} \mathscr{H}_{A K}^{k c}-\mathscr{H}_{K C}^{a k} \rho_{A K}^{k c}\right) ; \\
& \sum_{k} \rho_{A A}^{k k}=\mathscr{A} \\
& |A\rangle,|C\rangle,|K\rangle \in\left\{\left|I_{1}\right\rangle,\left|I_{2}\right\rangle, \ldots,\left|I_{m}\right\rangle\right\} .
\end{aligned}
$$

(3.5) ist ein Gleichungssystem für die verallgemeinerte Dichtematrix $\left(\rho_{A B}^{a b}\right)$; die Eigenwerte $\Omega_{A}$ des Quasiteilchenoperators $h$ sind nötig, um die Teilchenzahlbedingungen zu erfüllen. Bei einem Zwischenzustand reduziert sich die Hartree-Fock-Theorie mit Zwischenzuständen zur üblichen Hartree-Fock-Theorie, weil dann die Austauschterme bezüglich der $\mathscr{N}$-TeilchenZwischenzustände mit den direkten Termen zusammenfallen. Mit der Dichtematrix liegen alle Matrixelemente von Ein- und Zweiteilchen-Operatoren zwischen den Zuständen $\left|I_{1}\right\rangle,\left|I_{2}\right\rangle, \ldots,\left|I_{m}\right\rangle$ fest; so sind in unserer Hartree-Fock-Näherung die Energien des Vielteilchensystems die Erwartungswerte

$$
\begin{aligned}
& \langle A|H| A\rangle \\
& =\sum_{i_{1}, i_{1}} T^{i_{1} i_{1} i_{1}}\left\langle A\left|a_{i_{1}}^{\dagger} a_{i_{1}}\right| A\right\rangle \\
& +\frac{1}{4} \sum_{i_{2}, i_{1}, i_{1}, i_{2}} V^{i_{2} i_{1}, i_{2} i_{1}}\left\langle A\left|a_{i_{2}}^{\dagger} a_{i_{1}^{\prime}}^{\dagger} a_{i_{1}} a_{i_{2}}\right| A\right\rangle \\
& =\sum_{i_{1}, i_{1}} T^{i_{1} i_{1} i_{1}} \rho_{A A}^{i_{1} i_{1}^{\prime}}+\frac{1}{2} \sum_{i_{2}^{\prime}, i_{1}, i_{1}, i_{2}, K} V^{i_{2}^{i} i_{1}, i_{2} i_{1}} \rho_{A K}^{i_{2} i_{2}} \rho_{K A}^{i_{1} i_{1}} .
\end{aligned}
$$

Es gibt demnach zwei Möglichkeiten, die Energien des Vielteilchensystems zu berechnen: Gleichung (3.5) oder Gleichung (3.8). Da die Eigenwerte $\Omega_{A}$ die Rolle Lagrangescher Multiplikatoren spielen, sind die Konsistenzbeziehungen

$\Omega_{A}=\langle A|H| A\rangle$

nicht automatisch erfüllt. Obwohl sie sich, wie wir zeigen werden, leicht erfüllen lassen, ist es oft günstiger, darauf zu verzichten. Daher verstehen wir unter HartreeFock-Theorie mit Zwischenzuständen immer nur das Gleichungssystem (3.5). Dieser Verzicht hat nicht nur rechnerische Vorteile: Während in die Lagrangeschen Multiplikatoren nur die Strukturunterschiede der Zwischenzustände eingehen, kommt es bei den Erwartungswerten auf die tatsächliche Struktur an. Sie 
sind also um so ungenauer. je exponierter die Zwischenzustände liegen. Daher ist es leicht möglich, daß die Resultate von (3.5) durch (3.9) nicht verbessert werden.

Durch Gleichung (3.9) lassen sich die richtigen Lösungen von Gleichung (3.5) auswählen: Die Lösungen von (3.5) treten paarweise auf, weil mit $\rho_{A B}^{a b}$ auch

$\check{\rho}_{A B}^{a b}=\rho_{B A}^{a b}$

eine Lösung ist. Das Gleichungssystem (3.5) hat in $\check{\rho}$ die Gestalt

$$
\begin{aligned}
& -2\left(\Omega_{A}-\Omega_{B}\right) \check{\rho}_{A C}^{a c}=\sum_{k, K}\left(\check{\rho}_{A K}^{a k} \check{\mathscr{H}}_{K C}^{k c}-\check{\mathscr{H}}_{A K}^{a k} \check{\rho}_{K C}^{k c}\right. \\
& \left.+\check{\rho}_{K(}^{a k} \check{\mathscr{H}}_{A K}^{k c}-\check{\mathscr{H}}_{K C}^{a k} \check{\rho}_{A K}^{k c}\right) ; \quad \sum_{k} \check{\rho}_{A A}^{k k}=\mathscr{H} .
\end{aligned}
$$

Die beiden Lösungen liefern wegen

$$
\begin{aligned}
& \sum_{i_{1}, i_{1}} T^{i_{1} i_{1}} \grave{\rho}_{A 4}^{i_{1} i_{1}}+\frac{1}{2} \sum_{i_{2}, i_{1}, i_{1}, i_{2}, \Lambda} V^{i_{2} i_{1}, i_{2} i_{1}} \check{\rho}_{A K}^{i_{2} i_{2}} \check{\rho}_{\Lambda, 1}^{i_{1} i_{1}} \\
& =\sum_{i_{1}, i_{1}} T^{i_{1}^{\prime} i_{1}} \rho_{A A}^{i_{1} i_{1}^{\prime}}+\frac{1}{2} \sum_{i_{2}^{\prime}, i_{1}^{\prime}, i_{1}, i_{2}, K} V^{i_{2}^{\prime} i_{1}^{\prime}, i_{2} i_{1}} \rho_{A K}^{i_{2} i_{2}^{\prime}} \rho_{K A}^{i_{1} i_{1}^{\prime}}
\end{aligned}
$$

dieselben Energieerwartungswerte. Die zweite Lösung $\check{\rho}$ scheidet aus, da zu ihr die Energien $-\Omega_{A}$ und die Energieerwartungswerte $\langle A|H| A\rangle$ gehören, die Konsistenzbeziehungen (3.9) also nicht einmal näherungsweise erfüllt sind.

Aus (3.5) folgt eine Orthonormierungsbedingung für die Zwischenzustände $\left|I_{1}\right\rangle,\left|I_{2}\right\rangle, \ldots,\left|I_{m}\right\rangle$ :

$\left(\Omega_{A}-\Omega_{C}\right)\langle A|N| C\rangle=0, \quad$ d.h. $\langle A|N| C\rangle=\mathscr{A} \delta_{A C}$,

die die lineare Unabhängigkeit dieser Zustände garantiert. Diese wichtige Eigenschaft ist an das Auftreten der Austauschterme bezüglich der $\mathscr{N}$-Teilchen-Zwischenzustände in Gleichung (3.5) gebunden. Daher ist die Forderung $\langle A|N| C\rangle=0$ für $|A\rangle \neq|C\rangle$ [15] überflüssig.

\section{Lösung der Hartree-Fock-Theorie mit Zwischenzuständen durch doppelte Selbstkonsistenz}

\subsection{Die Struktur der verallgemeinerten Dichtematrix}

Wir geben in Abschnitt 4 ein Lösungsverfahren für das Gleichungssystem (3.5) und die Konsistenzbeziehung (3.9) an, die als Gleichungssysteme für die verallgemeinerte Dichtematrix $\left(\rho_{A B}^{a b}\right)$ aufzufassen sind. Ausgangspunkt unserer Überlegungen ist die Beobachtung, daß die verallgemeinerte Dichtematrix als hermitische Matrix aufgefaßt werden kann. Aus der Definitionsgleichung (3.6) folgt

$\rho_{A B}^{a b}=\rho_{B A}^{b a *}$.

Gleichung (4.1) bedeutet Hermitizität der verallgemeinerten Dichtematrix, wenn man sich die übereinander stehenden Indizes zu einem gemeinsamen Index zu- zusammengefaßt denkt. Die Schreibweise

$\rho^{(a)(B)}=\rho_{A B}^{a b}$

bringt das klarer zum Ausdruck; in ihr nimmt Gleichung (4.1) die Gestalt

$\rho^{\left({ }_{A}^{a}\right)\left(\begin{array}{l}b \\ B\end{array}\right)}=\rho^{(\stackrel{b}{B})(\underset{A}{a}) *}$

an. Da die verallgemeinerte Dichtematrix hermitisch ist, kann sie durch eine unitäre Matrix diagonalisiert werden:

$\rho_{A B}^{a b}=\sum_{1, T} U_{A T}^{a l} \rho_{D}^{\left(\frac{b}{T}\right)} U_{B T}^{h l *}$

In Matrixform lautet diese Gleichung

$\rho=U \rho_{D} U^{\dagger}$

Bei positiv-definierter Norm im Hilbert-Raum sind die Elemente der reellen Diagonalmatrix $\left(\rho_{D}^{(t)}\right)$ nach oben beschränkt; aus den Gleichungen (3.6) und (4.4) folgt

$$
\begin{aligned}
& \rho_{D}^{\left(\frac{t}{)}\right)}=\sum_{\substack{a, A \\
b, B}} U_{A T}^{a t *}\left\langle A\left|a_{b}^{\dagger} a_{a}\right| B\right\rangle U_{B T}^{b t} \\
& =1-\left(\sum_{a, A} U_{A T}^{a t *}\langle A| a_{a}\right)\left(\sum_{b, B} U_{B T}^{b t} a_{b}^{\dagger}|B\rangle\right) \leqq 1 .
\end{aligned}
$$

Es ist daher eine zu starke Einschränkung, $\rho$ als Projektor [15] anzusehen. Gleichung (4.5) ist nicht die einzige Einschränkung für die Eigenwerte der verallgemeinerten Dichtematrix. Da die Zwischenzustände . $/$-Teilchenzustände sind, folgt aus der Definitionsgleichung (3.6) die Spurbedingung

$\sum_{t . T} \rho_{D}^{\left(\frac{t}{T}\right)}=\sum_{a . A} \rho_{A A}^{a a}=m \cdot 1$.

Gleichung (4.6) ist eine notwendige Bedingung, die Nebenbedingungen $\sum_{a} \rho_{A A}^{a a}=\mathscr{A}$ zu erfüllen. Wegen (4.6) bleiben $m-1$ Nebenbedingungen übrig. Da in den Bewegungsgleichungen (3.5) nur die Differenzen $\Omega_{A}-\Omega_{C}$ der Lagrangeschen Multiplikatoren vorkommen, gibt es genau so viele linear unabhängige Differenzen wie Nebenbedingungen. Daher ist das Glejchungssystem (3.5) widerspruchslos.

Die Hermitizität der verallgemeinerten Dichtematrix hat die Hermitizität der verallgemeinerten HartreeFock-Matrix (3.7) zur Folge:

$\mathscr{H}_{A B}^{a b}=\mathscr{H}_{B A}^{b a *}$.

Wir bezeichnen $\left(\rho_{A B}^{a b}\right)$ nach (4.4) faktorisiert mit $\left(\rho_{A B}^{a b}\left(U, \rho_{D}\right)\right)$; ebenso bezeichnen wir $\left(\mathscr{H}_{A B}^{a b}\right)$ als Funktion von $U$ und $\rho_{D}$ mit $\left(\mathscr{H}_{A B}^{a b}\left(U, \rho_{D}\right)\right)$.

\subsection{Lösung der Hartree-Fock-Theorie mit Zwischenzuständen}

Wir zeigen, daß (3.5) nur als Gleichungssystem für die unitäre Matrix $U$ und nicht für die Diagonalmatrix $\rho_{D}$ 
aufzufassen ist; die Diagonalmatrix $\rho_{D}$ wird vielmehr durch Symmetrien und andere Randbedingungen des Vielteilchensystems bestimmt. $\mathrm{Zu}$ diesen Randbedingungen gehören auch die Konsistenzbeziehungen (3.9). Wir lösen hier das Gleichungssystem (3.5) für fest vorgegebene Diagonalmatrizen $\rho_{D}$; damit verzichten wir vorerst auf die Erfüllung der Konsistenzbeziehungen (3.9). Im nächsten Abschnitt werden wir die Auswahl geeigneter Diagonalmatrizen diskutieren.

Wir bringen das Gleichungssystem (3.5) in die Form

$\sum_{k, K}\left[\rho_{A K}^{a k}\left(\mathscr{H}_{K C}^{k c}+\delta^{k c} \delta_{K \mathcal{C}} \Omega_{\mathcal{C}}\right)-\left(\mathscr{H}_{A K}^{a k}+\delta^{a k} \delta_{A K} \Omega_{A}\right) \rho_{K C}^{k c}\right.$

$\left.+\rho_{K c}^{a k}\left(\mathscr{H}_{A K}^{k c}-\delta^{k c} \delta_{A K} \Omega_{A}\right)-\left(\mathscr{H}_{K C}^{a k}-\delta^{a k} \delta_{K C} \Omega_{C}\right) \rho_{A K}^{k c}\right]=0 ;$

$\sum_{k} \rho_{A A}^{k k}=\mathscr{N}$.

Wir könnten die Lösungsverfahren der HartreeBogoliubov-Theorie sofort übernehmen, wenn der Austauschterm bezüglich der $\mathscr{N}$-Teilchen-Zustände

$\mathscr{P}_{A C}^{a c}=\sum_{k, K}\left[\rho_{K C}^{a k}\left(\mathscr{H}_{A K}^{k c}-\delta^{k c} \delta_{A K} \Omega_{A}\right)\right.$

$\left.-\left(\mathscr{H}_{K C}^{a k}-\delta^{a k} \delta_{K C} \Omega_{C}\right) \rho_{A K}^{k c}\right]$

fehlte. Wir stellen jedoch leicht fest, daß die Lösungsverfahren der Hartree-Bogoliubov-Theorie [11] auf das Gleichungssystem

$$
\begin{aligned}
& \sum_{k, K}\left[\rho_{A K}^{a k}\left(\mathscr{H}_{K C}^{k c}+\delta^{k c} \delta_{K C} \Omega_{C}+Y_{K C}^{k c}\right)\right. \\
& \left.-\left(\mathscr{H}_{A K}^{a k}+\delta^{a k} \delta_{A K} \Omega_{A}+Y_{A K}^{a k}\right) \rho_{K C}^{k c}\right]=0 ; \\
& \sum_{k} \rho_{A \Lambda}^{k k}=\mathscr{N}
\end{aligned}
$$

anwendbar sind, wo $Y$ eine beliebige hermitische Matrix ist:

$Y_{A B}^{a b}=Y_{B A}^{b a *}$.

Wir bestimmen die unitäre Matrix $U$ durch Diagonalisieren der hermitischen Matrix $\left(\mathscr{H}_{A K}^{a k}\left(U, \rho_{D}\right)+\right.$ $\left.\delta^{a k} \delta_{A K} \Omega_{A}+Y_{A K}^{a k}\right)$ :

$\sum_{k, K}\left(\mathscr{H}_{A K}^{a k}\left(U, \rho_{D}\right)+\delta^{a k} \delta_{A K} \Omega_{A}+Y_{A K}^{a k}\right) U_{K B}^{k b}=U_{A B}^{a b} D^{\left(b^{b}\right)}$,

wobei die Lagrangeschen Multiplikatoren $\Omega_{A}$ so $\mathrm{zu}$ wählen sind, daß durch die unitäre Matrix $U$ die Nebenbedingungen

$\sum_{k} \rho_{A A}^{k k}\left(U, \rho_{D}\right)=\mathscr{N}$

erfüllt werden. Man prüft leicht nach, daß die durch (4.12) und (4.13) bestimmte Dichtematrix $\rho=U \rho_{D} U^{\dagger}$ eine Lösung des Hilfsgleichungssystems (4.10) ist. Da $Y$ beliebig gewesen ist, fragt man sich, ob es ein $Y$ gibt, so daß die Lösung von (4.10) auch Lösung von (4.8) ist. Wenn das der Fall ist, muß

$\sum_{k, K}\left[\rho_{A K}^{a k} Y_{K C}^{k c}-Y_{A K}^{a k} \rho_{K C}^{k c}\right]=\mathfrak{P}_{A C}^{a c}$ sein. Wir fragen also nach der Lösbarkeit des Gleichungssystems (4.14) für $Y$. Es ist in der Tat lösbar, wenn alle Matrixelemente des Hamilton-Operators (3.2) reell sind, d.h. bei Zeitumkehrinvarianz. Das trifft auf den weitaus größten Teil der Fälle zu. Dann ist auch $U$ reell, d.h. orthogonal. Der Beweis ist sehr einfach.

Da $\rho$ und $\mathscr{H}$ hermitisch sind, ist der Austauschterm bezüglich der $\mathscr{N}$-Teilchen-Zustände antihermitisch:

$\mathfrak{P}_{A C}^{a c}=-\mathfrak{H}_{C \cdot A}^{c a *}$.

Daher ist auch der mit der unitären Matrix $U$ transformierte Austauschterm antihermitisch:

$\mathfrak{I}^{\prime}=U^{\dagger} \mathfrak{A U} ; \quad \mathfrak{M}^{\prime}=-\mathfrak{M}^{\prime}$.

Die Diagonalelemente $\mathfrak{H}_{A A}^{a} a$ von $\mathfrak{H}^{\prime}$ sind also rein imaginär. d.h. bei Zeitumkehrinvarianz gleich 0 :

$\mathfrak{V}_{A A}^{\prime a a}=0$ bei Zeitumkehrinvarianz.

Wenn wir die mit der unitären bzw. orthogonalen Matrix $U$ transformierte Matrix $Y$ mit $Y^{\prime}$ bezeichnen:

$Y^{\prime}=U^{\dagger} Y U ; \quad Y^{\prime}=Y^{\prime \dagger}$,

nimmt Gleichung (4.14) die Gestalt

$\rho_{D} Y^{\prime}-Y^{\prime} \rho_{D}=\mathfrak{Q}^{\prime}$

an, d.h. die Gestalt

$\left(\rho_{D}^{(a)}-\rho_{D}^{(c)}\right) Y_{A C}^{\prime a c}=\mathfrak{H}_{A C}^{\prime a c}$.

Die Lösbarkeit des Gleichungssystems (4.14) entscheidet sich also am Gleichungssystem (4.19). Wir können ohne Beschränkung der Allgemeinheit annehmen, daß die Eigenwerte der verallgemeinerten Dichtematrix voneinander verschieden sind. Dann ist

$Y_{A C}^{\prime a c}=\frac{1}{\left(\rho_{D}^{(a)}-\rho_{D}^{(c)}\left(\begin{array}{c}c \\ c\end{array}\right)\right.} \mathfrak{I}_{A C}^{\prime a c} \quad$ für $\left(\begin{array}{l}a \\ A\end{array}\right) \neq\left(\begin{array}{l}c \\ C\end{array}\right)$.

Aus Stetigkeitsgründen sind auch die Matrixelemente $Y_{A C}^{\prime a c}$ bestimmt, wo $\rho_{D}^{(a)}=\rho_{D}^{(c)}$ für $\left(\begin{array}{l}a \\ A\end{array}\right) \neq\left(\begin{array}{l}c \\ C\end{array}\right)$ ist. Da bei Zeitumkehrinvarianz $\mathfrak{Q}_{A A}^{\prime a a}=0$ ist, lassen sich die Gleichungen (4.19) auch für $\left(\begin{array}{l}a \\ A\end{array}\right)=\left(\begin{array}{l}c \\ C\end{array}\right)$ lösen. Offensichtlich sind die Diagonalelemente $Y_{A A}^{\prime a}$ nicht eindeutig bestimmt; das ist verständlich, da die Kommutatorgleichung (4.14) keine eindeutig bestimmte Lösung hat. Damit haben wir nicht nur die Lösbarkeit des Gleichungssystems (4.14) bewiesen, sondern auch gleichzeitig ein Lösungsverfahren angegeben.

Die Lösungsverfahren für die Gleichungssysteme (4.10) und (4.14) lassen sich kombinieren zu einem Lösungsverfahren für die Hartree-Fock-Theorie mit Zwischenzuständen, d.h. für das Gleichungssystem (3.5). Da in allen bisher gerechneten Modellen $Y=0$ ist, lösen wir das Gleichungssystem (4.10) mit $Y=Y_{0}=0$. Mit dem so gewonnenen $\rho$ berechnen wir den Austauschterm 
bezüglich der $\mathscr{N}$-Teilchen-Zustände (4.9). Wenn er verschwindet, haben wir offensichtlich das ganze Gleichungssystem (3.5) gelöst. Verschwindet er jedoch nicht, so berechnen wir aus dem Gleichungssystem (4.14) ein $Y=Y_{1}$, wobei wir alle nicht eindeutig bestimmten Matrixelemente von $Y_{1}$ gleich 0 setzen. Danach beginnen wir wieder von vorn, indem wir das Gleichungssystem (4.10) mit $Y=Y_{1}$ lösen. Dieses Verfahren liefert eine Folge $Y_{0}, Y_{1}, Y_{2}, \ldots$, die gegen $Y$ konvergiert, weil alle Matrixelemente beschränkt sind. Dieses $Y$ tritt nach Konstruktion in (4.10) und (4.14) auf. Daher ist die Lösung von (4.10) auch Lösung von (4.8). Damit haben wir bei Zeitumkehrinvarianz die Hartree-Fock-Theorie mit Zwischenzuständen durch doppelte Selbstkonsistenz gelöst: Selbstkonsistenz für $\rho$ und Selbstkonsistenz für $Y$. Wir werden im übernächsten Abschnitt eine schematische Darstellung des Lösungsverfahrens der Hartree-Fock-Theorie mit Zwischenzuständen geben.

Nebenbei möchten wir noch feststellen, daß die Hartree-Fock-Theorie mit Zwischenzuständen symmetrischer ist als die Multi-Konfiguration-Methode von Fäßler $[16,17]$, da keine Spalte der unitären Matrix $U$ ausgezeichnet wird, was bei der Matrix der Entwicklungskoeffizienten nach Slater-Determinanten der Fall ist.

\subsection{Die Eigenwerte \\ der verallgemeinerten Dichtematrizen}

Wir haben in Abschnitt 4.2 die zu fest vorgegebenen Diagonalmatrizen $\rho_{D}$ gehörenden Lösungen des Gleichungssystems (3.5) gesucht. Da wir aber kein Prinzip zur Bestimmung der Eigenwerte $\rho_{D}^{(t)}$ kennen, machen wir den Vorschlag, die Eigenwerte $\rho_{0, D}^{(t)}$ der verallgemeinerten Schalenmodelldichtematrix $\rho_{0}=\left(\rho_{O A B}^{a b}\right) \mathrm{zu}$ nehmen. In der gewöhnlichen Hartree-Fock-Theorie z.B. sind sie entweder 0 oder 1 . Damit verzichten wir auf die Erfüllung der Konsistenzbeziehung (3.9). Jedoch begehen wir keinen großen Fehler: Die $\Omega_{A}$ sind als Lagrangesche Multiplikatoren in einem Gleichungssystem für die unitäre Matrix $U$ langsam veränderliche Funktionen der Eigenwerte, während die Erwartungswerte $\langle A|H| A\rangle$ schnell veränderliche Funktionen sind. Da man die Eigenwerte $\rho_{0, D}^{\left(\frac{t}{T}\right)}$ in physikalisch interessanten Fällen höchstens um $1 \%$ abändern muß, um die Konsistenzbeziehung (3.9) zu erfüllen, liefern die $\Omega_{A}$ die bessere Näherung für die Energien der Zwischenzustände $|A\rangle \in\left\{\left|I_{1}\right\rangle,\left|I_{2}\right\rangle, \ldots,\left|I_{m}\right\rangle\right\}$. Daher geben wir die Energiedifferenzen des $\mathscr{N}$-TeilchenSystems immer durch die $\Omega_{A}-\Omega_{C}$ wieder.

\subsection{Das Flußdiagramm für die Hartree-Fock-Theorie mit Zwischenzuständen}

Das folgende Diagramm gibt einen Überblick über das Lösungsverfahren der Hartree-Fock-Theorie mit
Zwischenzuständen, das wir in den vorhergehenden Abschnitten entwickelt haben.

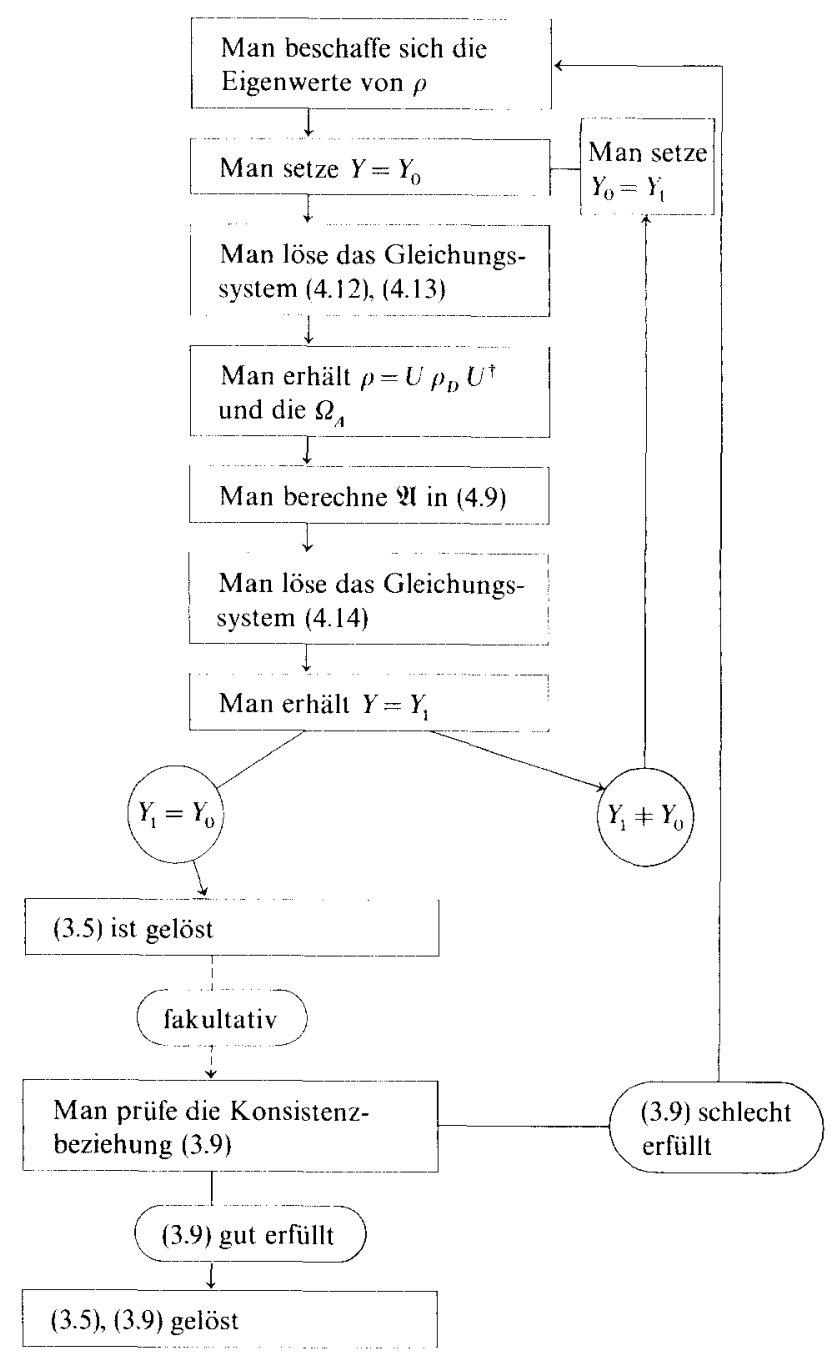

\section{Anwendung der Hartree-Fock-Theorie mit Zwischenzuständen auf das Kleinsche Rotations-Vibrationsmodell}

\subsection{Das Modell}

Wir testen die Hartree-Fock-Theorie mit Zwischenzuständen am Kleinschen Rotations-Vibrationsmodell [12-14], dessen Spektrum je nach den Werten der Kopplungskonstanten Rotations- oder Vibrationscharakter hat. Obwohl nur der Rotationsteil des Spektrums richtig wiedergegeben wird, lohnt sich die Anwendung auf dieses Modell, weil die Struktur der Gleichungen, der Einbau von Erhaltungssätzen und die Behandlung entarteter Niveaus gut zu überblicken sind.

Der Einteilchenraum des Kleinschen Rotations-Vibrationsmodells besteht aus zwei $(2 j+1)$-fach entarteten Schalen. Man bezeichnet die zugehörigen Fermion- 
Erzeugungs- und Vernichtungsoperatoren mit

$a_{m, \sigma}^{\dagger}, a_{m, \sigma} ; \quad m=-j,-j+1, \ldots, j ; \quad \sigma= \pm 1 ;$

$j$ ist halbzahlig.

Wir deuten die zweiwertige Variable $\sigma$ als Drehimpuls um eine feste Achse. Man erhält exakt lösbare Modelle, wenn man die Erzeugungs- und Vernichtungsoperatoren (5.1) zu den zehn Paarungs- und Multipoloperatoren der $R$ (5)-Lie-Algebra zusammenkoppelt:

$A_{0}^{\dagger}=\left(A_{0}\right)^{\dagger}=\frac{1}{\sqrt{2 \Omega}} \sum_{m}(-1)^{j-m} a_{m,+1}^{\dagger} a_{-m,-1}^{\dagger} ;$

$A_{+}^{\dagger}=\left(A_{+}\right)^{\dagger}=\frac{1}{2 \sqrt{\Omega}} \sum_{m}(-1)^{j-m} a_{m_{,}+1}^{\dagger} a_{-m,+}^{\dagger} ;$

$A_{-}^{\dagger}=\left(A_{-}\right)^{\dagger}=\frac{1}{2 \sqrt{\Omega}} \sum_{m}(-1)^{j-m} a_{m,-1}^{\dagger} a_{-m,-1}^{\dagger} ;$

$\tau_{+}=\left(\tau_{-}\right)^{\dagger}=\frac{1}{\sqrt{2 \Omega}} \sum_{m} a_{m,+1}^{\dagger} a_{m,-1} ;$

$\tau_{0}=\sum_{m}\left(a_{m,+1}^{\dagger} a_{m,+1}-a_{m,-1}^{\dagger} a_{m,-1}\right) ;$

$N=\sum_{m}\left(a_{m,+1}^{\dagger} a_{m,+1}+a_{m,-1}^{\dagger} a_{m,-1}\right) ; \quad 2 \Omega=2 j+1$.

Hier sind $\tau_{0}$ der Gesamtdrehimpulsoperator und $N$ der Teilchenzahloperator. Die Operatoren $\tau_{ \pm}, \tau_{0}$ bilden eine $S U(2)$-Lie-Unteralgebra mit dem CasimirOperator

$\vec{\tau}^{2}=\Omega\left(\tau_{+} \tau_{-}+\tau_{-} \tau_{+}\right)+\frac{1}{4} \tau_{0}^{2}$

und den Vertauschungsregeln

$\left[\tau_{0}, \tau_{ \pm}\right]= \pm 2 \tau_{ \pm} ; \quad\left[\tau_{+}, \tau_{-}\right]=\frac{\tau_{0}}{2 \Omega}$.

Das Kleinsche Rotations-Vibrationsmodell ist durch den Hamilton-Operator

$H=-G 2 \Omega A_{0}^{\dagger} A_{0}-F\left(\tau_{+} \tau_{\ldots}+\tau_{-} \tau_{+}\right)$,

die Summe einer Paarungs- und einer „QuadrupolQuadrupol"-Wechselwirkung, definiert. $H$ kommutiert nicht nur mit $\tau_{0}$ und $N$, sondern auch mit dem Operator $P$, der die Drehimpulsrichtung umkehrt:

$P \tau_{+} P^{-1}=\tau_{-} ; \quad P \tau_{0} P^{-1}=-\tau_{0} ; \quad$ usw.;

$P^{-1}=P^{\dagger}=P$.

Die Zustände $\left|\frac{\mathscr{N}}{2}, \frac{J_{0}}{2}, p\right\rangle$ seien die Eigenzustände von $H, \tau_{0}, N$ zu den Eigenwerten $E\left(\frac{\mathscr{N}}{2}, \frac{J_{0}}{2}, p\right), J_{0}, \mathcal{A}$.

Aus (5.6) läßt sich die Beziehung

$P\left|\frac{\mathscr{N}}{2}, \frac{J_{0}}{2}, p\right\rangle=\left|\frac{\mathscr{N}}{2},-\frac{J_{0}}{2}, p\right\rangle$ ableiten. Daher sind die Zustände, für die $J_{0} / 2 \neq 0$ ist, mindestens zweifach entartet. Wenn wir eine reelle Darstellung wählen, folgen aus (5.6) und (5.7) Symmetriebeziehungen zwischen den Matrixelementen der Operatoren (5.2) nach Art von

$$
\begin{aligned}
& \left\langle\frac{\mathscr{N}}{2}, \frac{J_{0}}{2}, p\left|\tau_{+}\right| \frac{\mathscr{N}}{2}, \frac{J_{0}}{2}-1, p^{\prime}\right\rangle \\
& =\left\langle\frac{\mathcal{A}}{2},-\frac{J_{0}}{2}, p\left|\tau_{-}\right| \frac{\mathscr{N}}{2},-\frac{J_{0}}{2}+1, p^{\prime}\right\rangle \\
& =\left\langle\frac{\mathscr{N}}{2}, \frac{J_{0}}{2}-1, p^{\prime}\left|\tau_{-}\right| \frac{\mathscr{N}}{2}, \frac{J_{0}}{2}, p\right\rangle \\
& =\left\langle\frac{\mathcal{H}}{2},-\frac{J_{0}}{2}+1, p^{\prime}\left|\tau_{+}\right| \frac{\mathscr{N}}{2},-\frac{J_{0}}{2}, p\right\rangle .
\end{aligned}
$$

In (5.8) haben wir schon die aus (5.4) folgenden Auswahlregeln

$$
\begin{aligned}
& \left\langle\frac{\mathscr{A}}{2}, \frac{J_{0}}{2}, p\left|\tau_{ \pm}\right| \frac{\mathcal{N}}{2}, \frac{J_{0}^{\prime}}{2}, p^{\prime}\right\rangle \\
& =\delta_{A, \ldots}, \delta_{J_{0}, J_{0} \pm 2}\left\langle\frac{\mathscr{N}}{2}, \frac{J_{0}}{2}, p\left|\tau_{ \pm}\right| \frac{\mathscr{N}}{2}, \frac{J_{0}}{2} \mp 1, p^{\prime}\right\rangle
\end{aligned}
$$

berücksichtigt. Der Vollständigkeit halber geben wir die Auswahlregeln für die Operatoren

$N_{ \pm}=\frac{1}{2}\left(N \pm \tau_{0}\right)$

an:

$\left\langle\frac{\mathscr{N}}{2}, \frac{J_{0}}{2}, p\left|N_{ \pm}\right| \frac{\mathscr{N}^{\prime}}{2}, \frac{J_{0}^{\prime}}{2}, p^{\prime}\right\rangle$
$=\delta_{,,, 1,} \delta_{J_{0}, J_{0}^{\prime}} \delta_{p, p^{\prime}} \frac{1}{2}\left(\mathscr{N} \pm J_{0}\right)$.

Da die Hartree-Fock-Theorie mit Zwischenzuständen nur den Rotationsteil des Spektrums richtig wiedergibt, muß die Übereinstimmung im Grenzfall $G=0$, wo das Spektrum ein reines Rotationsspektrum ist, besonders gut sein. In diesem Grenzfall kann $H$ in die Form

$\left.H\right|_{G=0}=-\frac{F}{4 \Omega}\left(4 \vec{\tau}^{2}-\tau_{0}^{2}\right)$

gebracht werden. Die Basisvektoren für die Darstellung von $S U$ (2) liefern die Eigenzustände von $\left.H\right|_{G=0}$ :

$\tau_{0}\left|\frac{\mathscr{V}}{2}, \frac{J}{2}, \frac{J_{0}}{2}\right\rangle=J_{0}\left|\frac{\mathscr{V}}{2}, \frac{J}{2}, \frac{J_{0}}{2}\right\rangle ;$

$\vec{\tau}^{2}\left|\frac{\mathscr{J}}{2}, \frac{J}{2}, \frac{J_{0}}{2}\right\rangle=\frac{J}{2}\left(\frac{J}{2}+1\right)\left|\frac{\mathscr{N}}{2}, \frac{J}{2}, \frac{J_{0}}{2}\right\rangle ;$

$\left.H\right|_{G=0}\left|\frac{\mathscr{V}}{2}, \frac{J}{2}, \frac{J_{0}}{2}\right\rangle=-\frac{F}{2 \Omega}\left(J(J+2)-J_{0}^{2}\right)\left|\frac{\mathscr{V}}{2}, \frac{J}{2}, \frac{J_{0}}{2}\right\rangle ;$

$0 \leqq J=\mathscr{A}, \mathscr{N}-4, \mathcal{A}-8, \ldots ;$

$J_{0}=-J,-J+2,-J+4, \ldots, J$. 
Man sieht sofort, daß bei gerader Teilchenzahl im Grundzustand $J=\mathscr{N} ; J_{0}=0$ und im zweifach entarteten, ersten angeregten Zustand $J=\mathscr{A} ; J_{0}= \pm 2$ sind. $\operatorname{lm}$ Grenzfall $G=0$ sind auch die Matrixelemente

$\sqrt{2 \Omega}\left\langle\frac{1}{2}, \frac{J}{2}, \frac{J_{0}}{2} \pm 1\left|\tau_{ \pm}\right| \frac{f}{2}, \frac{J}{2}, \frac{J_{0}}{2}\right\rangle$

$=\sqrt{\frac{J}{2}\left(\frac{J}{2}+1\right)-\frac{J_{0}}{2}\left(\frac{J_{0}}{2} \pm 1\right)}$

bekannt.

\subsection{Die Hartree-Fock-Gleichungen} mit Zwischenzuständen für das Modell

Wir setzen in die Gleichungen (3.5) und (3.9) der Hartree-Fock-Theorie mit Zwischenzuständen den speziellen Hamilton-Operator (5.5) des Kleinschen Rotations-Vibrationsmodells ein. Sie nehmen eine besonders einfache Form an, wenn wir voraussetzen, daß die Zwischenzustände $\left|I_{1}\right\rangle,\left|I_{2}\right\rangle, \ldots,\left|I_{m}\right\rangle$ in einem irreduziblen Darstellungsraum von $R(5)$ liegen. Dann können wir nämlich setzen

$$
\begin{aligned}
& \left\langle A\left|a_{m, \pm 1}^{\dagger} a_{n, \pm 1}\right| B\right\rangle=\frac{1}{2 \Omega}\left\langle A\left|N_{ \pm}\right| B\right\rangle \delta_{m n} ; \\
& \left\langle A\left|a_{m, \pm 1}^{\dagger} a_{n, \mp 1}\right| B\right\rangle=\frac{1}{\sqrt{2} \Omega}\left\langle A\left|\tau_{ \pm}\right| B\right\rangle \delta_{m n},
\end{aligned}
$$

und die Gleichungen (3.5) lauten falsche $\Omega$-Abhängigkeit haben $[13,14]$. Man verifiziert leicht die Orthonormierungsbedingung (3.13):

$\left\langle A\left|N_{+}\right| B\right\rangle+\left\langle A\left|N_{-}\right| B\right\rangle=1 \delta_{A B}$.

Mit ihr vereinfacht man die Konsistenzbeziehung (3.9) $\mathrm{zu}$

$$
\begin{aligned}
& \Omega_{A}=-F \frac{1}{2 \Omega}+\left(\frac{F}{2 \Omega^{2}}-\frac{G}{2 \Omega}\right) \sum_{K}\left\langle A\left|N_{+}\right| K\right\rangle\langle K|N,| A\rangle \\
& -\left(F+\frac{G}{2}\right) \sum_{K}\left(\left\langle A\left|\tau_{+}\right| K\right\rangle\left\langle K\left|\tau_{-}\right| A\right\rangle\right. \\
& \left.+\langle A|\tau \quad| K\rangle\left\langle K\left|\tau_{+}\right| A\right\rangle\right) .
\end{aligned}
$$

\subsection{Spezialisierung auf gerade Teilchenzahl} und drei $Z$ wischenzustände, den Grundzustand und den zweifach entarteten, ersten angeregten Zustand

\subsubsection{Einbau von Erhaltungssätzen} und Behandlung entarteter Niveaus

Wir haben die Eigenzustände von $H, \tau_{0}, N$ mit $\left|\frac{1}{2}, \frac{J_{0}}{2}, p\right\rangle$ bezeichnet. Wenn wir beabsichtigen, bei gerader Teilchenzahl die Eigenschaften des Grundzustands $\left|\frac{1}{2}, 0, p_{0}\right\rangle$ und des zweifach entarteten, ersten angeregten Zustands $\left|\frac{1}{2},+1, p_{+}\right\rangle ;\left|\frac{1}{2},-1, p_{\ldots}\right\rangle$ $\mathrm{zu}$ bestimmen, müssen wir als Zwischenzustände die

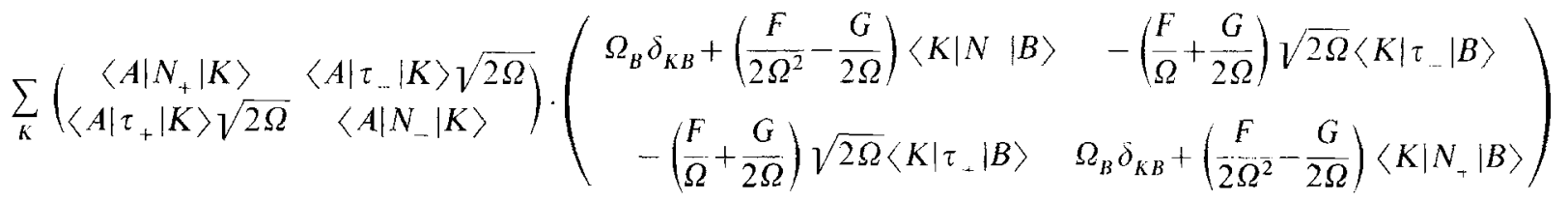

$$
\begin{aligned}
& =\sum_{K}\left(\begin{array}{cc}
\Omega_{A} \delta_{A K}+\left(\frac{F}{2 \Omega^{2}}-\frac{G}{2 \Omega}\right)\left\langle A\left|N_{-}\right| K\right\rangle & -\left(\frac{F}{\Omega}+\frac{G}{2 \Omega}\right) \sqrt{2} \bar{\Omega}\left\langle A\left|\tau_{-}\right| K\right\rangle \\
-\left(\frac{F}{\Omega}+\frac{G}{2 \Omega}\right) \sqrt{2 \Omega}\left\langle A\left|\tau_{+}\right| K\right\rangle & \Omega_{A} \delta_{A K}+\left(\frac{F}{2 \Omega^{2}}-\frac{G}{2 \Omega}\right)\left\langle A\left|N_{-}\right| K\right\rangle
\end{array}\right) \cdot\left(\begin{array}{cc}
\left\langle K\left|N_{+}\right| B\right\rangle & \left\langle K\left|\tau_{-}\right| B\right\rangle \sqrt{2} \Omega \\
\left\langle K\left|\tau_{+}\right| B\right\rangle \sqrt{2 \Omega} & \left\langle K\left|N_{. .}\right| B\right\rangle
\end{array}\right) ; \\
& \left\langle A\left|N_{+}\right| A\right\rangle+\left\langle A\left|N_{-}\right| A\right\rangle=\mathscr{H} ; \quad|A\rangle,|B\rangle,|K\rangle \in\left\{\left|I_{1}\right\rangle,\left|I_{2}\right\rangle, \ldots,\left|I_{m}\right\rangle\right\} .
\end{aligned}
$$

An Gleichung (5.16) fällt sofort auf, daß sie die Gestalt von Gleichung (4.10) mit $Y=0$ hat. Das Selbstkonsistenzproblem für $Y$ ist also von vornherein gelöst; es bleibt das Selbstkonsistenzproblem für die verallgemeinerte Dichtematrix. Weiter stellt man fest, daß der Vibrationsteil des Spektrums nicht richtig wiedergegeben wird, weil die zu $G$ proportionalen Terme die
Zustände

$$
\begin{aligned}
& |0\rangle=\left|\frac{1}{2}, 0, p_{0}\right\rangle ; \quad|+1\rangle=\left|\frac{4}{2},+1, p_{+}\right\rangle ; \\
& |-1\rangle=\left|\frac{1}{2},-1, p_{-}\right\rangle
\end{aligned}
$$


in das Gleichungssystem (5.16) aufnehmen. Es liefert allerdings nur Näherungen für diese Zustände, die nicht alle Quantenzahlen der exakten Zustände besitzen. Nach Konstruktion haben die Näherungszustände im allgemeinen keine feste Teilchenzahl; man kann nur den Erwartungswerten die richtige Teilchenzahl geben. Die $\tau_{0}$-Erhaltung läßt sich über die Auswahlregeln (5.9) und (5.11) für die Matrixelemente der Operatoren $\tau_{ \pm}$und $N_{ \pm}$berücksichtigen. Dabei zerfällt das Gleichungssystem (5.16):

$\rho_{+0} \mathscr{H}_{+0} \oplus \rho_{-0} \mathscr{H}_{-0} \oplus \rho_{+1} \mathscr{H}_{+1} \oplus \rho_{-1} \mathscr{H}_{-1}$

$=\mathscr{H}_{+0} \rho_{+0} \oplus \mathscr{H}_{-0} \rho_{-0} \oplus \mathscr{H}_{+1} \rho_{+1} \oplus \mathscr{H}_{-1} \rho_{-1} ;$

$\left\langle 0\left|N_{+}\right| 0\right\rangle+\left\langle 0\left|N_{-}\right| 0\right\rangle=\mathscr{N}$;

$\left\langle \pm 1\left|N_{+}\right| \pm 1\right\rangle+\left\langle \pm 1\left|N_{-}\right| \pm 1\right\rangle=\mathscr{N}$,

mit

$\rho_{ \pm 0}=\left\langle \pm 1\left|N_{ \pm}\right| \pm 1\right\rangle ;$

$\rho_{ \pm 1}=\left(\begin{array}{cc}\left\langle \pm 1\left|N_{\mp}\right| \pm 1\right\rangle & \sqrt{2} \Omega\left\langle \pm 1\left|\tau_{ \pm}\right| 0\right\rangle \\ \sqrt{2} \Omega\left\langle 0\left|\tau_{\mp}\right| \pm 1\right\rangle & \left\langle 0\left|N_{ \pm}\right| 0\right\rangle\end{array}\right) ;$

$\mathscr{H}_{ \pm 0}=\Omega_{ \pm 1}-\Omega_{0}+A\left\langle \pm 1\left|N_{\mp}\right| \pm 1\right\rangle$;

$\mathscr{H}_{ \pm 1}$

$=\left(\begin{array}{cc}\Omega_{ \pm 1}-\Omega_{0}+A\left\langle \pm 1\left|N_{ \pm}\right| \pm 1\right\rangle & B \sqrt{2 \Omega}\left\langle \pm 1\left|\tau_{ \pm}\right| 0\right\rangle \\ B \sqrt{2 \Omega}\left\langle 0\left|\tau_{\mp}\right| \pm 1\right\rangle & A\left\langle 0\left|N_{\mp}\right| 0\right\rangle\end{array}\right)$

und

$A=\left(\frac{F}{2 \Omega^{2}}-\frac{G}{2 \Omega}\right) ; \quad B=-\left(\frac{F}{\Omega}+\frac{G}{2 \Omega}\right)$.

Schließlich berücksichtigen wir die $P$-Erhaltung über die Symmetriebeziehungen (5.8). Das führt zu

$\rho_{-0}=\rho_{+0} ; \quad \rho_{-1}=\rho_{+1}$,

so da $\beta$ die Gleichung $\rho_{-0} \mathscr{H}_{-0} \oplus \rho_{-1} \mathscr{H}_{-1}=\mathscr{H}_{-0} \rho_{-0} \oplus$ $\mathscr{H}_{-1} \rho_{-1}$ die Gestalt der Gleichung $\rho_{+0} \mathscr{H}_{+0} \oplus \rho_{+1} \mathscr{H}_{+1}$ $=\mathscr{H}_{+0} \rho_{+0} \oplus \mathscr{H}_{+1} \rho_{+1}$ annimmt. Daraus folgt

$\Omega_{-1}=\Omega_{+1}$,

also die zweifache Entartung des ersten angeregten Niveaus. Nach Einbau aller Erhaltungssätze hat das Gleichungssystem (5.16) für die drei Zwischenzustände $|0\rangle,| \pm 1\rangle$ die einfache Gestalt

$\rho_{+0} \mathscr{H}_{+0} \oplus \rho_{+1} \mathscr{H}_{+1}=\mathscr{H}_{+0} \rho_{+0} \oplus \mathscr{H}_{+1} \rho_{+1} ;$

$2\left\langle 0\left|N_{+}\right| 0\right\rangle=\mathscr{.}^{\prime}$;

$\left\langle+1\left|N_{+}\right|+1\right\rangle+\left\langle+1\left|N_{-}\right|+1\right\rangle=\mathscr{N}$.

\subsubsection{Lösung}

Wir lösen das Gleichungssystem (5.23) mit den in Abschnitt 4 entwickelten Methoden. Es dient zur Be- stimmung der orthogonalen Matrix in der Zerlegung (4.4), wobei man die Eigenwerte der verallgemeinerten Dichtematrix als bekannt voraussetzt. Die Zerlegung von $\rho_{+0}$ und $\rho_{+1}$, ist sehr einfach:

$\rho_{+0} \equiv\left\langle+1\left|N_{+}\right|+1\right\rangle=1 \cdot q_{1} \cdot 1$;

$\rho_{+1} \equiv\left(\begin{array}{cc}\left\langle+1\left|N_{-}\right|+1\right\rangle & \sqrt{2} \Omega\left\langle+1\left|\tau_{+}\right| 0\right\rangle \\ \sqrt{2} \Omega\left\langle 0\left|\tau_{\ldots}\right|+1\right\rangle & \left\langle 0\left|N_{+}\right| 0\right\rangle\end{array}\right)$

$=\left(\begin{array}{cc}\cos \varphi & \sin \varphi \\ -\sin \varphi & \cos \varphi\end{array}\right)\left(\begin{array}{cc}q_{2} & 0 \\ 0 & q_{3}\end{array}\right)\left(\begin{array}{cc}\cos \varphi & -\sin \varphi \\ \sin \varphi & \cos \varphi\end{array}\right)$.

Da die Gleichung $\rho_{+0} \mathscr{H}_{+0}=\mathscr{H}_{+0} \rho_{+0}$ trivialerweise erfüllt ist, haben wir nur noch, analog zu (4.12), die zweireihige Matrix $\mathscr{H}_{+1}$ zu diagonalisieren. Dabei geht das Gleichungssystem (5.23) über in

$\left(\left(\Omega_{+1}-\Omega_{0}\right)\right.$

$\left.+A\left(q_{1}-\mathscr{N}+q_{2} \sin ^{2} \varphi+q_{3} \cos ^{2} \varphi\right)\right) \cos \varphi \sin \varphi$

$+B\left(-q_{2}+q_{3}\right)\left(\cos ^{2} \varphi-\sin ^{2} \varphi\right) \cos \varphi \sin \varphi=0 ;$

$\frac{\mathscr{N}}{2}=q_{2} \sin ^{2} \varphi+q_{3} \cos ^{2} \varphi ; \quad \frac{3 \cdot \mathscr{A}}{2}=q_{1}+q_{2}+q_{3}$.

Die letzte Gleichung ist die Spurbedingung (4.6). Das Gleichungssystem (5.25) hat die Lösung

$\Omega_{+1}-\Omega_{0}=\left(F\left(\frac{1}{\Omega}-\frac{1}{2 \Omega^{2}}\right)+G \frac{1}{\Omega}\right)\left(q_{1}-\frac{\mathscr{V}}{2}\right) ;$

$2 \Omega\left\langle+1\left|\tau_{+}\right| 0\right\rangle^{2}=\left(\mathscr{N}-q_{1}-q_{3}\right)\left(\frac{\mathscr{N}}{2}-q_{3}\right) ;$

$\left\langle+1\left|N_{-}\right|+1\right\rangle=\mathscr{N}-q_{1}$;

$\left\langle 0\left|N_{+}\right| 0\right\rangle=\frac{\mathscr{N}}{2}$.

Sie nützt wenig, wenn es nicht gelingt, sich die Eigenwerte $q_{1}, q_{2}, q_{3}$ zu beschaffen. $q_{1}$ ist bekannt, wenn man dem Matrixelement $\left\langle+1\left|N_{+}\right|+1\right\rangle$ den exakten Wert (5.11) gibt:

$q_{1}=\frac{1}{2}(\mathscr{N}+2)$.

Damit ist die Lösung (5.26) bis auf das Matrixelement $\left\langle+1\left|\tau_{+}\right| 0\right\rangle$ eindeutig bestimmt. Da die Hartree-FockTheorie mit Zwischenzuständen ohnehin nur den Rotationsteil des Spektrums richtig wiedergibt, darf man für $q_{2}, q_{3}$ die Eigenwerte der Matrix $\rho_{+1}$ im Grenzfall $G=0$ wählen. Nach (5.14) ist

$\left.\rho_{+1}\right|_{\substack{\text { exakt } \\ G=0}}=\left(\begin{array}{cc}\frac{1}{2}(\mathscr{N}-2) & \sqrt{\frac{\mathscr{N}}{2}\left(\frac{\mathscr{N}}{2}+1\right)} \\ \sqrt{\frac{\mathscr{N}}{2}\left(\frac{\mathcal{N}}{2}+1\right)} & \frac{1}{2} \mathscr{N}\end{array}\right)$,

so daß

$q_{2}=\mathscr{N} ; \quad q_{3}=-1$ 
sind. Wenn wir diese Werte in die Gleichungen (5.26) einsetzen, erhalten wir

$$
\begin{aligned}
& \Omega_{+1}-\Omega_{0}=\frac{F}{\Omega}\left(1-\frac{1}{2 \Omega}\right)+\frac{G}{\Omega} ; \\
& 2 \Omega\left\langle+1\left|\tau_{+}\right| 0\right\rangle^{2}=\frac{\mathscr{N}}{2}\left(\frac{\mathscr{N}}{2}+1\right) ; \\
& \left\langle+1\left|N_{-}\right|+1\right\rangle=\frac{1}{2}(\mathscr{N}-2) ; \\
& \left\langle 0\left|N_{+}\right| 0\right\rangle=\frac{\mathscr{A}}{2} .
\end{aligned}
$$

Im Grenzfall $G=0$ stimmen diese Werte mit Ausnahme der Energiedifferenz $\Omega_{+1}-\Omega_{0}$ mit den exakten Werten überein; diese unterscheidet sich von dem exakten Wert (5.13) $\left.\left(\Omega_{+1}-\Omega_{0}\right)\right|_{\substack{\text { exakt } \\ G=0}}=F / \Omega$ um den Korrekturterm $-\frac{F}{2 \Omega^{2}}$. Alles in allem gibt die HartreeFock-Theorie mit Zwischenzuständen den Rotationsteil des Spektrums ausgezeichnet wieder.

\subsubsection{Die Konsistenzbeziehung}

für die Erwartungswerte von $H$ in den Zwischenzuständen $|0\rangle,| \pm 1\rangle$

Wenn man nachprüft, ob die Lösung der HartreeFock-Theorie mit Zwischenzuständen die Konsistenzbeziehung (3.9) erfüllt, stößt man auf Schwierigkeiten. Mit den Matrixelementen (5.26) findet man für die Erwartungswerte (5.18) die Ausdrücke

$$
\begin{aligned}
& \langle 0|H| 0\rangle=-F \frac{N}{2 \Omega}+\left(\frac{F}{2 \Omega^{2}}-\frac{G}{2 \Omega}\right) \frac{\mathcal{H}^{2}}{4} \\
& -\left(F+\frac{G}{2}\right) 2 \frac{1}{2 \Omega}\left(\mathcal{H}-q_{1}-q_{3}\right)\left(\frac{\mathscr{N}}{2}-q_{3}\right) \\
& \langle \pm 1|H| \pm 1\rangle=-F \frac{N}{2 \Omega}+\left(\frac{F}{2 \Omega^{2}}-\frac{G}{2 \Omega}\right) q_{1}\left(\mathscr{N}-q_{1}\right) \\
& -\left(F+\frac{G}{2}\right) \frac{1}{2 \Omega}\left(\mathcal{H}-q_{1}-q_{3}\right)\left(\frac{\mathscr{N}}{2}-q_{3}\right) .
\end{aligned}
$$

Es fällt sofort auf, daß sich die letzten Terme der beiden Ausdrücke um den Faktor 2 unterscheiden. Er würde auch im zweiten Ausdruck nicht fehlen, wenn wir mehr Zwischenzustände genommen hätten. Die Erwartungswerte sind also sehr empfindlich gegen Beimischung anderer Zustände. Sie sind um so ungenauer, je höher die Zwischenzustände liegen. Die Lagrangeschen Multiplikatoren weisen diesen Mangel nicht auf, da in sie nur die Strukturunterschiede der Zustände eingehen, und nicht, wie in die Erwartungswerte, deren tatsächliche Struktur.

Damit ist verständlich, daß im Rotationsteil des Spektrums die Lage des Grundzustandes durch die
Gleichungen (5.31), (5.27), (5.29) gut wiedergegeben wird, während die Lage des ersten angeregten $\mathrm{Zu}$ stands aus Gleichung (5.30) folgt. Der Erwartungswert

$\langle 0|H| 0\rangle=-\frac{F}{4 \Omega} \cdot \mathcal{A}\left(A+4-\frac{A}{2 \Omega}\right)-\frac{G}{4 \Omega} \cdot \mathcal{A}(1+1)$

stimmt im Grenzfall $G=0$ gut mit dem exakten Wert (5.13)

$\left.\langle 0|H| 0\rangle\right|_{\substack{\text { exakt } \\(i \ldots 0}}=-\frac{F}{4 \Omega} \cdot H(\cdot 1+2)$

uiberein.

\section{Schluß}

Die Erweiterung der Neuen Tamm-Dancoff-Methode durch Einführung von Zwischenzuständen führt zu einer Fülle neuer Beziehungen in der nicht-relativistischen und relativistischen Vielteilchentheorie. Wir haben hier nur das nach der Hartree-Fock- und der Hartree-Bogoliubov-Theorie nächst einfache, nichtrelativistische Gleichungssystem untersucht: die Hartree-Fock-Theorie mit $Z$ wischenzuständen. Wir haben sie auf das Kleinsche Rotations-Vibrationsmodell angewendet, wo sie mit drei Zwischenzuständen den Rotationsteil des Spektrums gut wiedergibt. Wenn wir versucht hätten, den Rotations- und den Vibrationsteil gleichzeitig zu erfassen, hätten wir die HartreeBogoliubov-Theorie mit Zwischenzuständen anwenden müssen. Das bleibt einer späteren Arbeit überlassen. Trotzdem hoffen wir, daß die einfachen, durchsichtigen und erfolgreichen Modellrechnungen den Anstoß zur Behandlung realistischerer Vielteilchensysteme mit der Hartree-Fock-Theorie mit Zwischenzuständen geben. Der Rechenaufwand wird nicht die Grenzen des Gewohnten überschreiten. Ganz allgemein kann man sagen, daß die Hartree-FockTheorie mit zwei Zwischenzuständen einfacher als die Hartree-Bogoliubov-Theorie ist. Man wird daher bei kleineren Vielteilchensystemen die Hartree-Fock-Gleichungen mit zehn nicht miteinander entarteten Zwischenzuständen noch lösen können.

Die neue Tamm-Dancoff-Methode mit Zwischenzuständen ist ein kovariantes Näherungsverfahren. So ist sie für die Heisenbergsche nicht-lineare Spinortheorie [18] von Nutzen: Die relativistische Version der Hartree-Fock-Theorie mit Zwischenzuständen ermöglicht eine selbstkonsistente Berechnung von Vakuum und Bosonenzuständen. Andererseits können wir auch mesonische Variablen berücksichtigen [19] und so einen neuen Zugang zu dem heute sehr aktuellen Problem der Pion-Kondensation [20] schaffen.

A. Friederich und W. Gerling danken dem Bundesministerium für Forschung und Technologie und der Deutschen Forschungsgemeinschaft für die Finanzierung dieser Arbeit. 


\section{Literatur}

1. Migdal, A.B.: Nuclear theory: the quasiparticle method, p. 35. New York, Amsterdam: W.A. Benjamin, Inc. 1968

2. Friederich, A., Gerling, W., Bleuler, K.: Z. Naturforsch. 30a, $142(1975)$

3. Gorkov, L. P.: J. Exptl. Theoret. Phys. 7, 505 (1958)

4. Brenig, W., Wagner, H.: Z. Physik 173, 484 (1963)

5. Friederich, A.: Z. Physik 223, 152 (1969)

6. Bleuler, K., Friederich, A., Petry, H.-R., Schütte, D.: In: Quanten und Felder, pp. 267-277. Braunschweig: Vieweg u. Sohn 1971

7. Dürr, H.P., Wagner, F.: Nuovo Cimento 46, 223 (1966)

8. Kerman, A. K., Klein, A.: Phys. Rev. 132, 1326 (1963)

9. Kerman, A. K., Klein, A.: Phys. Rev. 138B, 1323 (1965)

10. Klein, A., Calenza, L., Kerman, A.K.: Phys. Rev. 140B, 245 (1965)

11. Baranger, M.: In: Cargèse lectures in theoretical physics 1962, pp. 61-89, ed. by M. Lévy. New York: W.A. Benjamin, Inc. 1963

12. Chattopadhyay, P.K., Krejs, F., Klein, A.: Phys. Lett. 42 B, 315 (1972)

13. Dasso, C., Krejs, F., Klein, A., Chattopadhyay, P.K.: Nucl. Phys. A 210, $429(1973)$
14. Chattopadhyay, P.K., Klein, A., Krejs, F.: Nucl. Phys. A 229, 509 (1974)

15. Kamlah, A., Meyer, J.: Z. Physik 211, 293 (1968)

16. Fäßler, A., Plastino, A.: Z. Physik 220, 88 (1969)

17. Fäßler, A., Schmid, K.W., Plastino, A.: Nucl. Phys. A 174, 26 (1971)

18. Heisenberg, W.: Einführung in die einheitliche Feldtheorie der Elementarteilchen, pp. 66-80. Stuttgart: Hirzel 1967

19. Novozhilov, Y.V., Tulub, A.V.: The method of functionals in the quantum theory of fields. New York: Gordon and Breach 1961

20. Migdal, A. B.: Nucl. Phys. A 210, 421 (1973)

\author{
A. Friederich \\ W. Gerling \\ Institut für Theoretische Kernphysik \\ der Universität Bonn \\ Nußallee 14-16 \\ D-5300 Bonn \\ Bundesrepublik Deutschland
}

